\title{
Risk based facility location by using fault tree analysis in disaster management ${ }^{\text {is }}$
}

\author{
İbrahim Akgün ${ }^{\mathrm{a}, *}$, Ferhat Gümüşbuğa ${ }^{\mathrm{b}}$, Barbaros Tansel ${ }^{\mathrm{c}}$ \\ a Abdullah Gül University, Department of Industrial Engineering, Kayseri, Turkey \\ ${ }^{\mathrm{b}}$ Turkish Army Military Academy, Institute of Defense Sciences, Ankara, Turkey \\ ${ }^{\mathrm{C}}$ Bilkent University, Department of Industrial Engineering, Bilkent, Ankara, Turkey
}

\section{A R T I C L E I N F O}

Article history:

Received 19 August 2013

Accepted 20 April 2014

Processed by Salazar-Gonzalez

Available online 14 May 2014

Keywords:

Location

Risk

Fault tree analysis

Vulnerability

Disaster management

p-Center risk model

\begin{abstract}
A B S T R A C T
Determining the locations of facilities for prepositioning supplies to be used during a disaster is a strategic decision that directly affects the success of disaster response operations. Locating such facilities close to the disaster-prone areas is of utmost importance to minimize response time. However, this is also risky because the facility may be disrupted and hence may not support the demand point(s). In this study, we develop an optimization model that minimizes the risk that a demand point may be exposed to because it is not supported by the located facilities. The purpose is to choose the locations such that a reliable facility network to support the demand points is constructed. The risk for a demand point is calculated as the multiplication of the (probability of the) threat (e.g., earthquake), the vulnerability of the demand point (the probability that it is not supported by the facilities), and consequence (value or possible loss at the demand point due to threat). The vulnerability of a demand point is computed by using fault tree analysis and incorporated into the optimization model innovatively. To our knowledge, this paper is the first to use such an approach. The resulting non-linear integer program is linearized and solved as a linear integer program. The locations produced by the proposed model are compared to those produced by the p-center model with respect to risk value, coverage distance, and covered population by using several test problems. The model is also applied in a real problem. The results indicate that taking the risk into account explicitly may create significant differences in the risk levels.
\end{abstract}

(c) 2014 Elsevier Ltd. All rights reserved.

\section{Introduction}

Schulz [1] defines a disaster as "an occurrence of widespread severe damage, injury or loss of life or property with which a community cannot cope and during which the society undergoes severe disruption" and Disaster Management (DM) as "the range of activities designed to maintain control over disaster and emergency situations and to provide a framework for helping at-risk persons to avoid or recover from the impact of the disaster. DM deals with situations before, during and after a disaster". The activities in the context of DM are generally considered in four phases: mitigation, preparedness, response, and recovery (e.g., [2-4]). Coppola [4] defines these phases as follows: "Mitigation involves reducing or eliminating the likelihood or the consequences of a hazard or both. Mitigation seeks to treat the hazard such that it impacts society to a lesser degree. Preparadness

\footnotetext{
This manuscript was processed by Associate Editor Salazar-Gonzalez.

* Corresponding author.

E-mail addresses: ibrahim.akgun@agu.edu.tr (İ. Akgün), fgumusbuga@gmail.com (F. Gümüșbuğa), barbaros@bilkent.edu.tr (B. Tansel).
}

involves equipping people who may be impacted by a disaster or who may be able to help those impacted with the tools to increase their chance of survival and to minimize their financial and other losses. Response involves taking action to reduce or eliminate the impact of disasters that have occurred or currently occurring, in order to prevent further suffering, financial loss, or a combination of both. Relief, a term commonly used in international disaster management, is one component of response. Recovery involves returning victims' lives back to a normal state following the impact of disaster consequences. The recovery phase generally begins after the immediate response had ended and can persist for months or years thereafter". The activities related to mitigation and preparedness, i.e., pre-disaster phase, are considered as Risk Management while the activities related to response and recovery, post-disaster phase, are considered as Crisis Management (e.g., [5])

Coppola [4] points out that response and recovery alone are not effective means of managing disasters if they are performed in the absence of a comprehensive regimen of preparadness and mitigation activities. Coppola [4] also points out that there is a shift towards risk reduction-based disaster management. This is also 
emphasized in the framework developed at the World Confence on Disaster Reduction held in Japan in 2005 [6]. Three strategic goals outlined in that framework are as follows:

1. The more effective integration of disaster risk considerations into sustainable development policies, planning and programming at all levels, with a special emphasis on disaster prevention, mitigation, preparedness, and vulnerability reduction,

2. The development and strengthening of institutions, mechanisms and capacities at all levels, in particular at the community level, that can systematically contribute to building resilience to hazards,

3. The systematic incorporation of risk reduction approaches into the design and implementation of emergency preparedness, response, and recovery programmes in the reconstruction of affected communities.

In this context, this paper studies one of the problems in the pre-disaster phase from a risk management perspective. Specifically, the study investigates the problem of locating the facilities that are used for pre-positioning stocks needed in the first stages of a disaster relief operation. The paper explicitly considers the risk that a demand point (probable disaster point) may not get service from the located facilities and tries to choose the locations of the facilities to minimize the maximum risk.

Balcik and Beamon [7] describe the general flow of resources to the disaster-affected areas as shown in Fig. 1. The resource requirements in the assessment phase are minimal while the resource requirements in the deployment phase increase dramatically. The need for resources stabilizes in the sustainment phase and decreases in the reconfiguration phase. The length and importance of each phase varies depending on the characteristics of the disaster and the characteristics of the affected areas. However, Balcik and Beamon [7] state that "the speed of relief operations during the first days of the disaster significantly affects the lives of many people, threatened by the disaster. The ability of a relief organization to mobilize its resources during assessment and deployment phases is critical to the success of disaster response".

One strategy that has gained importance and adopted by the relief organizations to enhance their emergency response capacity and hence to respond to a disaster effectively is to pre-position supplies. This strategy is important because, as Balcik and Beamon [7] state, most of the critical supplies arriving at the disaster areas are sourced from relief organizations' pre-positioned stocks. Similarly,
Jahre and Heigh [8] state that the performance of a disaster relief operation is much dependent on the level of preparedness.

Thomas and Mizushima [9] define pre-positioning as "the storage of inventory at or near the location at which it will be used". Although the importance of locating resources close to the disaster area for faster delivery of supplies to the affected people cannot be overemphasized, this also poses an important risk; the facilities (and hence supplies) themselves may be damaged or inaccessible due to the disaster(s). This study takes this fact into account in determining the locations of facilities. This is achieved implicitly as a part of the vulnerability of a demand point that depends on the locations of facilities (whether the demand point is covered or not) and computed by using Fault Tree Analysis (FTA). The structure in the FTA is incorporated into an optimization model. The resulting model is a non-linear mixed-integer programming model that aims to minimize the maximum risk that a demand point may be exposed to. The non-linear model is linearized and solved as a linear integer program.

The rest of the paper is organized as follows. Section 2 reviews the literature. Section 3 gives the risk definition used in the paper and Section 4 explains the fault tree analysis and how to compute the vulnerability of a demand point. Section 5 and Section 6 give the $p$-center risk and $p$-center models, respectively. Section 7 explains how the models are solved. Section 8 analyzes the model by considering several factors. Section 9 defines an application of the proposed model. Section 10 concludes the paper.

\section{Literature review}

DM has mostly been related to the social research and hence there is a rich literature in this context. The survey papers by Altay and Green [3] and Galindo and Batta [10] indicate that DM has gained importance in the last two decades in OR/MS (Operations Research/Management Science) research as well. Altay and Green [3] review 109 papers from 1980 to 2005 while Galindo and Batta [10] review 156 papers from 2006 to 2010 . They classify the papers with respect to several criteria and identify potential research directions for the OR/MS community. In this paper, we consider only the optimization models with a focus on facility location in DM and on reliable facility location in general.

Caunhye et al. [11] review optimization models in emergency logistics. They categorize the studies into two main categories: (1) facility location and (2) relief distribution and casualty transportation. They state that most facility location models combine

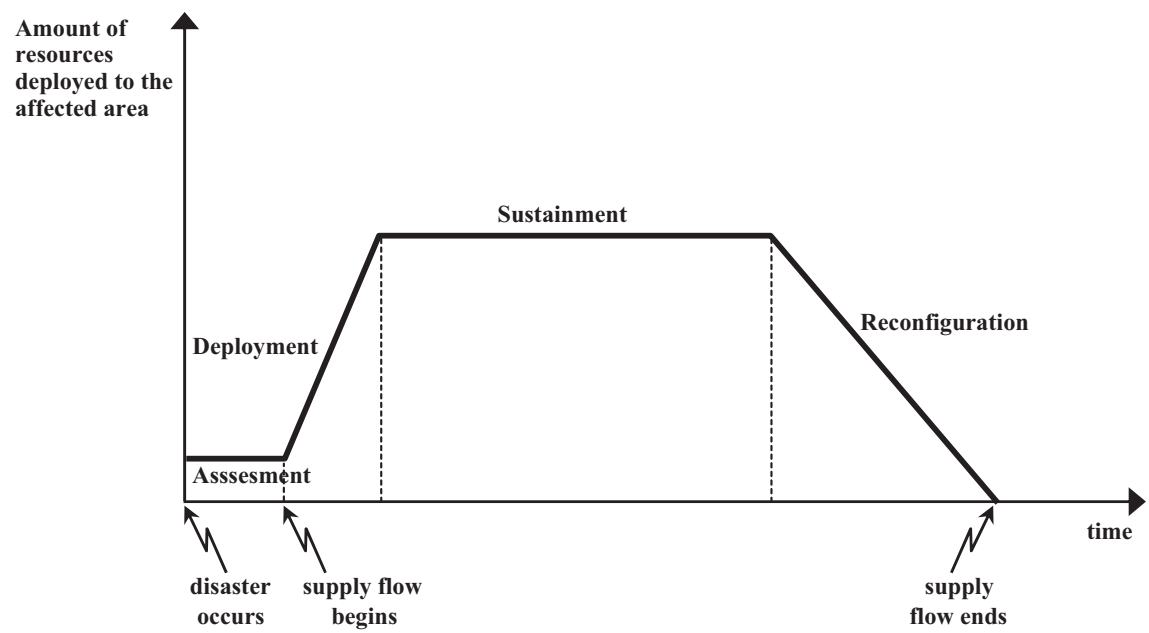

Fig. 1. Relief mission life cycle (Balcik and Beamon [7]). 
the process of location with stock-prepositioning, evacuation, and relief distribution and that the only location-alone models are published by Jia et al. ([12,13]) and Dessouky et al. [14]. Those models are formulated as maximal covering location models with multiple-coverage requirements. Dessouky et al. [14] additionally include a stochastic vehicle routing model to perform relief distribution. In this paper, a location-alone model is developed, i.e., decisions regarding the inventory levels of prepositioned stocks are not considered.

Belardo et al. [15] and Balcik and Beamon [7] combine location and stock prepositioning. They develop models that try to locate the facilities such that maximum demand is covered. They assign stock to the facilities in order to meet demands and do not consider the routing of stock through the network. Balcik and Beamon [7] include budget and response time requirements as well.

According to the classification by Caunhye et al. [11], other facility location models, e.g., Charnes et al. [16], McCall [17], and Wilhelm and Srinivasa [18], combine relief distribution and stockprepositioning mainly for cost minimization. Unlike other studies, Duran et al. [19] develop a time-minimizing model. Rawls and Turnquist [20] include ordering costs, holding costs, and the possibility of post-disaster damage to stock. They also state that most of the research has concentrated on post-disaster management and little research has been conducted on the topic of a priori planning, i.e., what resources should be stockpiled at which locations so that emergency response is most effective in the event that it is needed.

The idea of reliable facility location is not new. Daskin [21] considers reliability in the context of a maximum covering location model. The author takes into account the fact that a facility may not serve a demand because it is busy serving other demands. This is achieved by assigning predetermined, identical probabilities to all facilities. Drezner [22] considers new variants of the classical $p$-median and $p$-center location problems. Unlike the standard definitions, each facility may fail to serve its allocated customers and these customers may then have to be served by an alternate source. Each facility has a known failure probability that is a function of the facility design and independent of the location. The modified objective for the p-median problem contains the expected cost of each customer being served by its closest facility, its next closest, etc. The modified $p$-center problem chooses the locations of the $p$ facilities to minimize the maximum travel time where the travel time is based on the worst case scenario of $q$ facilities failing. Lee [23] considers the same modified $p$-median formulation as in Drezner [22] as well as a similar algorithm. Snyder and Daskin [24] model the cost of using backup facilities when facilities fail. The authors also present a reliability-based formulation of the uncapacitated fixed charge location problem. They assume that the failure probabilities of the facilities are independent. Berman et al. [25] extend the results in Drezner [22] and focus on structural results. Failure probabilities are not assumed to be identical but failures at different locations are again assumed to be independent. Berman et al. [26] study a class of location models where facilities are not perfectly reliable and failures may be correlated. They analyze problems with median and center objectives under complete and incomplete customer information regarding the state of facilities. They derive closedform analytical results for a 2 -facility problem on a unit segment, with customer demand distributed uniformly over the segment.

Reliable facility location is also considered in the context of DM. Rawls and Turnquist [20] consider the possibility that supply locations and/or roadways may be damaged or destroyed by using a scenario-based approach in a cost minimization model combining relief distribution and stock-prepositioning. A probability of occurence is assigned to each scenario where disruptions to the infrastructure and supplies are represented by capacity reductions.
If demand at a node is not met in a scenario or there is excess supply, several costs are incurred and incorporated into their model. Ukkusuri and Yushimoto [27] model the pre-positioning of supplies as a location routing problem. They maximize the probability that all the demand points can be served by a service location given fixed link/node failure probabilities and a specified budget constraint. Campbell and Jones [28] examine the decision of where to preposition supplies in preparation for a disaster and how much to preposition at a location. They derive equations for determining optimal stocking quantity and the total expected costs associated with delivering to a demand point from a supply point. They claim that their paper is the first to consider both risk and inventory levels without the use of scenarios. They assign probabilities to supply points to reflect the risk of being destroyed or made inaccessible. The types of costs and decisions considered are close to those in Rawls and Turnquist [20].

The literature indicates that uncertainties regarding the infrastructure are handled either by assigning probabilities or by defining scenarios. Galindo and Batta [10] state two drawbacks regarding the scenario-based approaches: (1) scenarios generally do not comprehend all the possible outcomes and (2) researchers generally assume the set of scenarios as a given input and they do not offer an efficient, systematic, and reliable way to define them. The authors point out that a more appropriate path would be to study the potential outcomes of a disaster through a solid probabilistic analysis.

One of the research directions offered by Galindo and Batta [10] is to incorporate coverage probabilities based on infrastructure reliabilities (vulnerabilities) while considering the locations of facilities. In this paper, we deviate from the current literature and address this issue by using one of the risk analysis tecniques, namely FTA.

\section{Definition of risk}

In this paper, we minimize the maximum risk that a demand point is not serviced due to disruptions to the located facilities. Although there are different definitions of risk, we use the one given by Willis et al. [29]. Even though the aforementioned definition is used in the context of terrorism risk, it can be adapted in other contexts as well. According to [29], risk is composed of three components, threat, vulnerability, and consequence. Threat is the probability that a specific target is attacked in a specific way during a specified time period. In our case, threat is the probability that a disaster (e.g., earthquake) occurs. Vulnerability is the probability that damages (where damages may involve fatalities, injuries, property damage, or other consequences) occur, given a specific attack type, at a specific time, on a given target. In our case, the vulnerability is the probability that damages occur at a demand point because it is not served on time by the located facilities. Vulnerability is computed by using FTA. Consequence is the expected magnitude of damage (e.g., deaths, injuries, or property damage), given a specific attack type, at a specific time, that results in damage to a specific target. In our case, the number of people living in an area (city) is considered to be the magnitude of the damage. Risk is then defined as the anticipated consequences over some period of time to a defined set of targets, resulting from a defined set of threats. Risk can be determined as a product of threat, vulnerability, and consequence.

\section{Fault tree analysis and vulnerability of a demand point}

FTA has been mostly used in engineering for reliability or hazard analysis (e.g., Rahman et al. [30] and Zhang et al. [31]). It is 
a deductive reasoning method used to identify failures prior to their occurrence, to analyze accidents, and/or as investigative tools to pinpoint failures. It is deductive in the sense that it starts with a general top event or output event and develop down through the branches to specific input events that must occur in order for the output to be generated. The top event for a fault tree is a negative event. Each event is analyzed by asking, "How could this happen?". In answering this question, the primary causes and how they interact to produce an undesired event are identified. This logic process continues until all potential causes have been identified. Throughout this process, a tree diagram is used to record the events as they are identified. Tree branches stop when all events leading to the negative event are complete.

The fault tree is constructed by using several symbols that represent various events and describe relationships. In the following, we only explain the ones used in the context of this study. AND gate is used when the output event will occur only if all of the input events exist simultaneously. OR gate is used when the event will occur if only one or any combination of the input events exists. Rectangle and circle are two symbols used to represent different types of events. A rectangle represents a negative main event that can be broken down into further input events. It can be located anywhere in the tree. It is only the rectangle that can have a logic gate and input events. A circle represents a base event in the tree and is found at the bottom tiers of the tree. A base event is not broken down into further events.

After the tree is constructed, the probabilities are assigned to the base events and the probability of the top event is computed in a bottom-up approach by multiplying (AND gates) and summing over (OR gates) probabilities of the events until the the top event is reached. For further information on FTA, see Vesely et al. [32].

Now, we explain how we develop the fault tree in our study on the sample network in Fig. 2. Suppose that all nodes are demand points and that the facilities can be located at all nodes. Assume that the vulnerability of demand point $A$ is to be computed. Thus, the top event $T$ is identified as the event that demand point $A$ is not supported by the located facilities. The top event may occur if none of the facilities located at the nodes $\mathrm{A}, \mathrm{B}$, and $\mathrm{C}$ can support the demand point. Notice that it is not known a priori where the facilities will be located and hence an input event is defined for each possible location and connected to the top event with an AND gate. From now on, we will proceed with node B. The part of the resulting fault tree for node $\mathrm{B}$ is given in Fig. 3 by using the same coding system to name the events.

We define the event that the facility at node B cannot support demand point $A$ as the main event $B$. Then, three events leading to event B are identified: (1) the event BTA that a facility is not located at node B, (2) the event BTK that a facility is located at node $B$ but node $B$ does not cover the demand point (whether a facility location covers a demand point or not is determined based on a predetermined coverage distance $K$ ), and (3) the event BTE that a facility is located at node $B$ and the demand point is covered; however, the facility is disrupted and hence inaccessible, i.e., supplies at location B are not usable. These three events (BTA, $\mathrm{BTK}$, and BTE) are connected to the input event B via an OR gate.

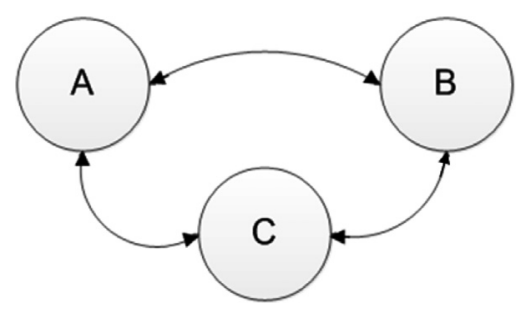

Fig. 2. Sample network.

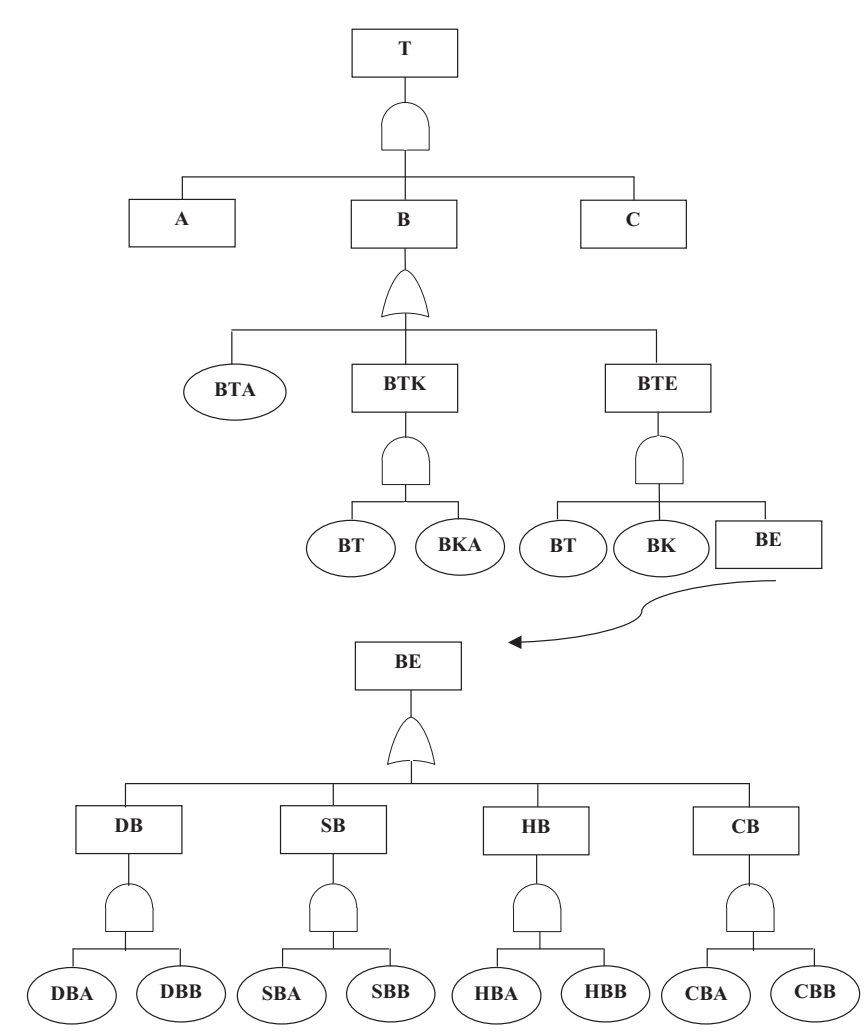

Fig. 3. The fault tree for three facility locations. The structure of the tree for main events $\mathrm{A}$ and $\mathrm{C}$ are the same as the one for main event $\mathrm{B}$.

Of these three events, the first one BTA is a base event and hence is not broken down into further events. The second event BTK is broken down into two more base events with an AND gate: (1) the event BT that a facility is located at node B and (2) the event BKA that the facility at node $B$ does not cover the demand point $A$. The third event BTE is broken down into three events via an AND gate: (1) the event BT that a facility is located at node B, (2) the event BK that the facility at node B covers the demand point, and (3) the event BE that the facility at node $B$ is disrupted and hence inaccessible. Of these three events, the first two (BT and BK) are base events. There may be several events leading to the disruption of the facility at node B, i.e., the main event BE. In this paper, four events are defined: (1) the event DB that an earthquake causes disruption, (2) the event SB that a flood causes disruption, (3) the event $\mathrm{HB}$ that a landslide causes disruption, and (4) the event $C B$ that an avalanche causes disruption. These four events are connected to the event BE via an OR gate. All of these four events are broken down into two base events similarly. For $C B$, the events are defined as follows: (1) the event CBA that there is an avalanche at node $B$ and (2) the event $C B B$ that there occurs a damage (disruption) that prevents access to the facility at node $B$ given an avalanche occurs. We remark that the analysis can be extended to include other events. For example, the vulnerability of the roads connected to the facility location, the vulnerability of the facility itself due to design, or the susceptibility of the facility to a terrorist attack may be considered. For the purposes of this study, we prefer to stop our analysis at this point.

The aforementioned tree structure is repeated for all demand points and then the vulnerability of each demand point, i.e., the probability of the top event for each demand point, is determined. However, notice that the locations of the facilities are not known a priori but the vulnerability of a demand point is dependent on where the facilities are located. In this regard, the probability of a top event cannot be computed directly by multiplying (AND gate) and summing (OR gate) probabilities; the facility location decisions 
are to be incorporated into the computation. The vulnerability of a demand point $j$ can be formulated as follows:

$v_{j}=\prod_{i \in I}\left\{\left(1-x_{i}\right)+\left[\left(1-a_{i j}\right) x_{i}\right]+a_{i j} e_{i} x_{i}\right\} \quad j \in J$

In the formulation (1), $I$ is the set of nodes that are candidates for facility location and $J$ is the set of demand nodes. $x_{i}$ is a binary variable that takes on the value of 1 if a facility is located at node $i$ and 0 otherwise. $a_{i j}$ is a parameter that is 1 if demand node $j$ is covered by the facility at node $i$. Assuming that the coverage is expressed in terms of shortest path lengths $d_{i j}$ and that $K$ is a predetermined coverage distance, $a_{i j}=1$ if $d_{i j} \leq K$ and $a_{i j}=0$ otherwise. $e_{i}$ is the probability that facility at node $i$ is disrupted and/or inaccesible, i.e., the probability of event $\mathrm{BE}$ in Fig. 3. Notice that $e_{i}$ is computed by using the part of the fault tree leading to BE.

The terms in (1) are derived from the fault tree. The first term in the equation represents the event that a facility is not located at a candidate point, e.g., the event BTA. The second term represents the event that a facility is located at the candidate point but it does not cover the demand point, e.g., BTK. Finally, the third term represents the event that a facility is located at a candidate point and it covers the demand point; however, the facility is disrupted and hence inaccessible, e.g, BTE. These are the events that lead to the main event that a facility located at at node cannot serve the demand point.

Each term in (1) represents the vulnerability of a demand point resulting from the aforementioned events for a specific facility location. For example, if a facility is not located, then the first term is 1 and the other terms are 0 meaning that the demand point is $100 \%$ vulnerable. If a facility is located but it does not cover the demand point, then the first and third terms are 0 and the second term is 1 and hence the demand point is $100 \%$ vulnerable. If a facility is located and it covers the demand point, then the first and second terms are 0 and the third term is $e_{i}$ meaning that the vulnerability of the demand point results only from the disruption of the facility for some reason, e.g., natural events. To sum up, the vulnerability of each demand point resulting from a specific location takes on a value between 0 and 1 as required for a probability value. The overall vulnerability of the demand point is computed by multiplying individual vulnerabilities resulting from candidate facility locations, which is in accordance with the AND gate in the fault tree. In other words, when a demand point is covered by more than one facility, its vulnerability decreases accordingly.

\section{5. p-center risk model}

We now give the $p$-Center Risk Model ( $p$-CR). The model is named as given because the maximum risk that a demand point is exposed to is minimized. $p$-center type models are considered more appropriate for situations where the equity is important (e.g., Lu and Sheu [33] and Bell et al. [34]), as is the case in a disaster management environment.

In addition to the definitions given in the previous section, we define $p$ as the number of facilities to locate, $c_{j}$ as the consequence (a value or loss, e.g., the number of people living) at demand point $j$ and $t_{j}$ as the probability that a threat (e.g., an earthquake) occurs at demand point $j$. Defining $R$ as the maximum risk that a demand node is exposed to, $p$-CR can be formulated as follows.

\subsection{Model p-CR: p-center risk model}

$$
R^{*}=\operatorname{Min}_{x, R} R
$$

s.t

$$
R \geq c_{j} t_{j} \prod_{i \in I}\left\{\left(1-x_{i}\right)+\left[\left(1-a_{i j}\right) x_{i}\right]+a_{i j} e_{i} x_{i}\right\} \quad j \in J
$$

$\sum_{i \in I} x_{i}=p$

$$
x_{i} \in\{0,1\} \quad i \in I
$$

Notice that the risk term on the right-hand-side of (3) is the same as the one defined earlier, i.e., the risk is the multiplication of consequence $c_{j}$, threat $t_{j}$, and the vulnerability $v_{j}=\prod_{i \in I}\left\{\left(1-x_{i}\right)+\right.$ $\left.\left[\left(1-a_{i j}\right) x_{i}\right]+a_{i j} e_{i} x_{i}\right\}$. The vulnerability term is exactly the same as Eq. (1) and used to compute the vulnerabilities of demand points depending on the values of the decision variable $x_{i}$. Thus, objective function (2) together with constraints (3) minimizes the maximum risk that demand points are exposed to. Constraints (4) require that exactly $p$ facilities be located. Constraints (5) define the integrality requirements for decision variables.

$p$-CR is not required to cover all demand points. Of course, covering all demand points is desirable. However, this may not be possible with given $p$ and $K$. Even though it is possible to cover all demand points, not covering some demand points may produce better risk values. For example, if covering a demand point with high population and high threat value more than once decreases its vulnerability significantly, the model may prefer not to cover some demand points with low population and low threat values in order to decrease the overall risk value.

We remark once again that $v_{j}$, the vulnerability of demand point $j$, changes depending on the locations of the facilities and is determined by the model endogeneously. This value can be computed only for a fixed set of facility locations and varies depending on the disruption probabilities of the facility locations as well as the number of facilities that cover the demand point. So, this is not the same as assigning a preset (risk) probability to a demand point (or to a facility location), which is mostly done in the literature.

\section{Vertex p-center model}

The coverage distance $K$ needed to determine $a_{i j}$ values in $p$-CR is obtained by solving the vertex $p$-Center $(p-C)$ model. $p$ - $C$ is also used to compare the results of $p-C R$ as will be explained later. The objective in $p-C$ is to find locations of $p$ facilities so that all demand points are covered and the maximum distance between a demand node and the nearest facility (coverage distance) is minimized. Because the objective is in minimax form, it is mostly applicable to emergency cases. The formulation used in our study is due to Hakimi [35]. In the formulation, $y_{i j}$ is a binary variable that takes on the value of 1 if the demand point $j$ is assigned to the facility located at node $i$ and the value of 0 otherwise. Defining $Z$ as the maximum distance between a demand point and the nearest facility, the formulation is as follows.

\subsection{Model p-C: $p$-center model}

In addition to (4) and (5);

$$
Z^{*}=\operatorname{Min}_{x, y, Z} Z
$$

s.t. $Z \geq \sum_{i} d_{i j} y_{i j} \quad j \in J$

$\sum_{i} y_{i j}=1 \quad j \in J$

$y_{i j} \leq x_{i} \quad i \in I, j \in J$

$y_{i j} \in\{0,1\} \quad i \in I, j \in J$ 
Objective function (6) together with constraints (7) minimizes the maximum distance between a demand point and the nearest facility. Constraints (8) ensure that each demand point be assigned to a single facility. Constraints (9) guarantee that allocation of a demand point can be made only to open facilities. Constraints (10) define the integrality requirement for the decision variables.

In some cases, considering demand-weighted distances may be more appropriate. This requirement can be incorporated into the model $p$-C by multiplying the right-hand sides of constraints (7) with the weights of the demand points. The resulting model is the weighted vertex $\mathrm{p}$-center problem $(p-C W)$. In this study, the weight of a demand point $j$ is set to $c_{j}$, i.e., the population.

\section{Solution of the models}

$p$-CR is a nonlinear optimization model with binary variables and hence is difficult to solve. However, it can be linearized and solved as a linear integer program.

We linearize $p$-CR in three steps. In the first step, we take the natural logarithm of constraints (3). This gives us constraints (11). Because $\ln$ terms except $\ln \left(c_{j} t_{j}\right)$ in (11) include variables, the resulting set of constraints is still nonlinear. In the second step, we linearize the $\ln$ term on the right-hand-side by utilizing the observation that the inner part of the $\ln$ term takes the value of 1 when $a_{i j}=0$ (irregardless of whether $x_{i}=0$ or $x_{i}=1$ ) or $x_{i}=0$ (irregardless of whether $a_{i j}=0$ or $a_{i j}=1$ ) and $e_{i}$ when $a_{i j}=1$ and $x_{i}=1$. The resulting values are 0 and $\ln _{i}$ in the first and second cases, respectively. We can obtain the same values by moving $x_{i}$ out of the $\ln$ term and rearranging the inner part as given in (12). When $a_{i j}=0$ and $x_{i}=0$, the resulting value is 0 and when $a_{i j}=1$ and $x_{i}=1$, the resulting value is $\ln e_{i}$, as required.

$\ln R \geq \ln \left(c_{j} t_{j}\right)+\sum_{i \in I} \ln \left\{\left(1-x_{i}\right)+\left[\left(1-a_{i j}\right) x_{i}\right]+a_{i j} e_{i} x_{i}\right\} \quad j \in J$

$\ln R \geq \ln \left(c_{j} t_{j}\right)+\sum_{i \in I} \ln \left(1-a_{i j}+a_{i j} e_{i}\right) x_{i} \quad j \in J$

In the third and final step of the linearization, we use the fact that minimizing a monotonic function of a variable is equivalent to minimizing the variable itself. In this regard, we replace $\underset{x, R}{\operatorname{Min}} R$ in $p$-CR with $\underset{x, R}{\operatorname{Min}} \ln R$. Moreover, by setting $R_{1}=\ln R$, we come up with a linear integer program, say $p$-CRL, composed of (4) and (5) and (13) and (14). The optimal objective function value of $p$-CR is $R^{*}=e^{R_{1}^{*}}$.

\subsection{Model p-CRL:linearized $p-C R$}

In addition to (4) and (5);

$R_{1}^{*}=\operatorname{Min}_{x, R_{1}} R_{1}$

$R_{1} \geq \ln \left(c_{j} t_{j}\right)+\sum_{i \in I} \ln \left(1-a_{i j}+a_{i j} e_{i}\right) x_{i} \quad j \in J$

Both $p$-CRL and $p$-C are coded in GAMS ([36]) and solved by CPLEX 12.1 ([37]) on a PC with 3 GB RAM and 2.40 Intel Core i3 CPU. All problem instances are solved to optimality in a matter of seconds for both $p$-CRL and $p$-C.

\section{Analysis of the proposed model $p$-CR}

To assess and gain insight about the results of $p$-CR, we define and solve a set of test problems. The problems are defined on a complete network where the nodes represent the cities (demand points and candidate facility locations) in Turkey. The distances between the cities are taken from the distance table prepared by the General Directorate of Highways. The population of each city is accepted as the value (consequence), $c_{j}$. The probability that an earthquake occurs in city is used as the threat. The threat probability of a city, $t_{j}$, is determined based on the earthquake risk zone that the city belongs to. The five risk zones are determined by the Turkish Catastrophe Insurance Pool (TCIP) taking Turkey's Earthquake Regions Map of the Ministry of Environment and Urban Planning as the basis. The risk zones are assigned the probabilities of $0.8,0.6,0.4,0.2$, and 0.0 from the first to the fifth, respectively. The probability that a facility at location $i$ cannot be reached, $e_{i}$, is computed as described in the fault tree by using the occurence probabilities of natural disasters and the probability that a devastating damage occurs given the associated natural disaster occurs. The former probabilities are obtained based on the risk zones as defined for the threat probability while the latter probabilities are taken from Gökçe et al. [38].

To see the effect of taking the risk into account explicitly, we consider risk values, covered populations, coverage distances, and facility locations obtained by solving $p-C(p-C W)$ and $p-C R$. We follow the steps of the procedure given in Fig. 4 to obtain the results. In the first step, $p-C$ is solved and coverage distance $K^{p-C}=Z^{*}$ and facility locations $x_{i}^{p-C}$ are saved. Because all demand points are required to be covered, the covered population by $p-C$ is $C^{p-C}=\Sigma_{j} c_{j}$. In the second step, $p$-CR is solved by using $K^{p-C}$ in determining the values of $a_{i j}$. Facility locations $x_{i}^{p-C R}$ and the risk value $R^{p-C R}=R^{*}$ are saved. The coverage distance $K^{p-C R}$ and covered population $C^{p-C R}$ are determined by postprocessing the

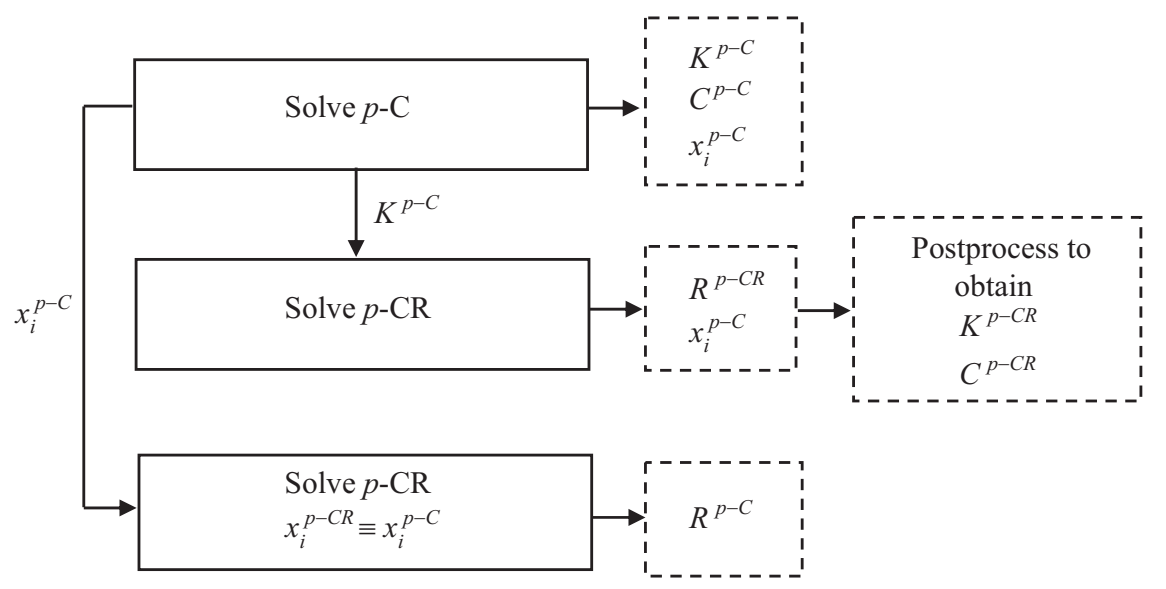

Fig. 4. Procedure to determine results of $p-C$ and $p-C R$. 
solution. $K^{p-C R}$ is the maximum distance between a demand point and the nearest located facility. Remember that covering the whole population is not required in $p-C R . C^{p-C R}$ is the population covered by the located facilities within a distance of $K^{p-C}$. So, $C^{p-C R}$ is obtained by summing over the populations of the cities that are within a distance of $K^{p-C}$ from a located facility. In the third step, the risk value $R^{p-C}$ generated by the solution of $p-C$ is computed by solving the $p$-CR with the facility locations set to $x_{i}^{p-C}$. When $p-C W$ is solved instead of $p$-C, the same procedure is used except that $p$-C replaced is with $p$-CW. Because different coverage distances are obtained for $p-C$ and $p-C W$, the results for p-CR change accordingly.

Table 1 (Table 2) gives the risk values, covered populations, and coverage distances of $p-C(p-C W)$ and $p-C R$ for $p$ changing between 1 and 10. Fig. 5 shows the results graphically. Fig. 6 depicts a sample solution and indicates the locations of the cities mentioned in the manuscript on the earthquake risk map of Turkey.

\subsection{Risk values}

For all problem instances in both unweighted and weighted cases, the risk values of $p-C R, R^{p-C R}$, are better (lower) than those of $p-C(p-C W), R^{p-C}\left(R^{p-C W}\right)$. This is actually expected but what is more important is the relative magnitude of the difference. The results indicate that the difference between the risk values varies between 2.5 times to 14.2 times with an average of about 8.2 times for $p-C$ and from 3.2 times to 30.4 times with an average of about 11.5 times for $p$-CW. To put the risk values into a meaningful context, the results imply that the expected losses (in terms of population) may be up to 8.2 (11.5) times more on the average when the risk of a demand point that is not supported by the located facilities is not considered explicitly.

Table 1

The results of $p-C$ and $p-C R$ for several parameters (unweighted case).

\begin{tabular}{|c|c|c|c|c|c|c|}
\hline \multirow[t]{2}{*}{$p$} & \multicolumn{3}{|l|}{$p-C$} & \multicolumn{3}{|l|}{$p-\mathrm{CR}$} \\
\hline & $R^{p-C}$ & $K^{p-C}$ & $C^{p-C}$ & $R^{p-C R}$ & $K^{p-C R}$ & $C^{p-C R}$ \\
\hline 1 & 424,182 & 1048 & $73,722,988$ & 169,673 & 1105 & $73,287,268$ \\
\hline 2 & 615,064 & 636 & $73,722,988$ & 158,378 & 1133 & $60,180,820$ \\
\hline 3 & 593,855 & 541 & $73,722,988$ & 94,772 & 796 & $69,817,550$ \\
\hline 4 & 572,646 & 504 & $73,722,988$ & 44,539 & 624 & $71,469,693$ \\
\hline 5 & 615,064 & 426 & $73,722,988$ & 57,535 & 754 & $70,192,687$ \\
\hline 6 & 572,646 & 377 & $73,722,988$ & 41,570 & 609 & $71,221,630$ \\
\hline 7 & 572,646 & 349 & $73,722,988$ & 40,364 & 434 & $72,343,460$ \\
\hline 8 & 296,927 & 318 & $73,722,988$ & 59,568 & 408 & $70,862,590$ \\
\hline 9 & 129,233 & 305 & $73,722,988$ & 40,587 & 434 & $71,273,770$ \\
\hline 10 & 466,600 & 276 & $73,722,988$ & 49,854 & 474 & $71,225,168$ \\
\hline
\end{tabular}

Table 2

The results of $p$-CW and $p$-CR for several parameters (weighted case).

\begin{tabular}{|c|c|c|c|c|c|c|}
\hline \multirow[t]{2}{*}{$p$} & \multicolumn{3}{|l|}{$p-\mathrm{CW}$} & \multicolumn{3}{|l|}{$p-\mathrm{CR}$} \\
\hline & $R^{p-C W}$ & $K^{p-C W}$ & $C^{p-C W}$ & $R^{p-C R}$ & $K^{p-C R}$ & $C^{p-C R}$ \\
\hline 1 & 551,436 & 1701 & $73,722,988$ & 148,464 & 2023 & $71,192,279$ \\
\hline 2 & 110,287 & 1473 & $73,722,988$ & 13,574 & 1105 & $73,722,988$ \\
\hline 3 & 33,025 & 1175 & $73,722,988$ & 1086 & 1105 & $73,722,988$ \\
\hline 4 & 38,530 & 821 & $73,722,988$ & 11,877 & 796 & $73,722,988$ \\
\hline 5 & 16,492 & 815 & $73,722,988$ & 2957 & 701 & $73,722,988$ \\
\hline 6 & 63,961 & 566 & $73,722,988$ & 7486 & 509 & $73,722,988$ \\
\hline 7 & 29,820 & 624 & $73,722,988$ & 1853 & 534 & $73,722,988$ \\
\hline 8 & 39,760 & 557 & $73,722,988$ & 2811 & 552 & $73,722,988$ \\
\hline 9 & 21,123 & 556 & $73,722,988$ & 1601 & 509 & $73,722,988$ \\
\hline 10 & 125,982 & 414 & $73,722,988$ & 10,932 & 414 & $73,722,988$ \\
\hline
\end{tabular}

The risk values of $p$-CR are better in the weighted case because coverage distances are higher than those in the unweighted case. In $p$-CW, the objective function value increases as the distance between a demand point with high population and its closest facility increases. This causes facility locations to be close to demand points with high population in different parts of the network. However, this produces coverage distances $K^{p-C W}$ significantly higher than $K^{p-C}$ for demand points with low population. Given higher coverage distances, $p$-CR can find facility locations that give better risk values with the same number of facilities. Actually, $p-\mathrm{CW}$ also produces better risk values than $p-C$ for all cases except for $p=1$. For $p=1, p$-CW locates the facility in Kocaeli (neihgboring İstanbul in the first earthquake zone and with the highest population constituting about $18 \%$ of the whole population) whose disruption probability is close to the highest value. The resulting risk value turns out to be the risk value of Istanbul. For $p=1, p-C$ locates the facility in Kayseri almost in the center of Turkey to minimize the distance. The resulting risk value turns out to be the risk value of Istanbul again. However, because the disruption probability of Kayseri is lower than that of Kocaeli, the resulting risk value is lower. Notice that even though the risk values are improved for both $p$-CW and $p$-CR in the weighted case, the improvement for $p$-CR is higher resulting in larger relative differences in the risk values. So, both cases indicate that considering risk explicitly has a value.

\subsection{Coverage distances and population coverages}

For all problem instances in the unweighted case, the coverage distance of $p-C R, K^{p-C R}$, is higher than that of $p-C, K^{p-C}$. Specifically, the ratio of $K^{p-C R}$ to $K^{p-C}$ changes from 1.05 to 1.78 with an average of 1.46 . That is, $p$-CR prefers not to cover some demand points within the coverage distance of $K^{p-C}$ to obtain better risk values. The percentage of the whole population covered by $p-C R$ within $K^{p-C}$ changes from $81.6 \%$ to $99.4 \%$ with an average of $95.2 \%$. To clarify, consider the case for $p=4$ in Table 1 . The solutions of $p-C$ and $p$-CR are depicted on the earthquake risk map of Turkey in Fig. 6. The locations of $p-C$ and $p-C R$ are represented by $M$ and $R$, respectively. The facility locations of $p-C$ cover all 81 demand points and hence the whole population with $K^{p-C}=504 \mathrm{~km}$. On the other hand, the facility locations of $p$-CR can cover about $97 \%$ of the whole population, i.e., $71,469,693$ out of $73,722,988$, within a distance of $504 \mathrm{~km}$. p-CR prefers not to cover nine demand points, whose population constitutes about $3 \%$ of the whole population, within this distance to obtain better risk values. The uncovered demand points are represented by $U$ in Fig. 6 . The maximum distance $K^{p-C R}$ turns out to be $624 \mathrm{~km}$ as shown in Fig. 6. The population of the demand point with the maximum distance to a facility is just $0.34 \%$ of the whole population.

For the weighted case, $K^{p-C R}$ is lower than (equal to) $K^{p-C W}$ for eight (one) instances out of 10 . For these instances, the ratio of $K^{p-C R}$ to $K^{p-C W}$ changes from 0.75 to 1 with an average of 0.91 . For the remaining one instance, the ratio is 1.19. Even though this result may seem counterintuitive at first glance, it is actually expected. As explained above, $p$-CW locates the facilities close to demand points with high population in certain parts of the network producing high coverage distances. In $p$-CR, the distance between a demand point and its closest facility is not a factor taken into account. What is important in $p-C R$ is whether a demand point is within the coverage distance or not; a facility covering the most populated demand point may be placed at a location $K$ distance units away from the demand point, which is not possible in $p$-CW. This flexibility of determining the locations may result in better coverage distances for $p$-CR. The exact locations of the facilities are dependent on the distribution of the disruption probabilities, threat probabilities, and population 
a

\section{unweighted case}

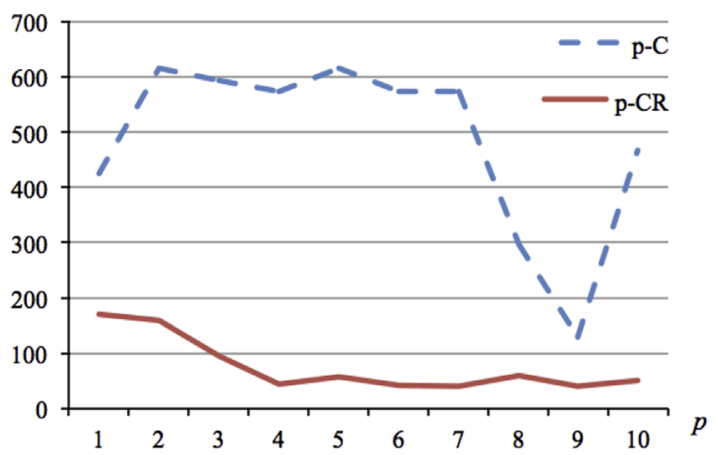

C

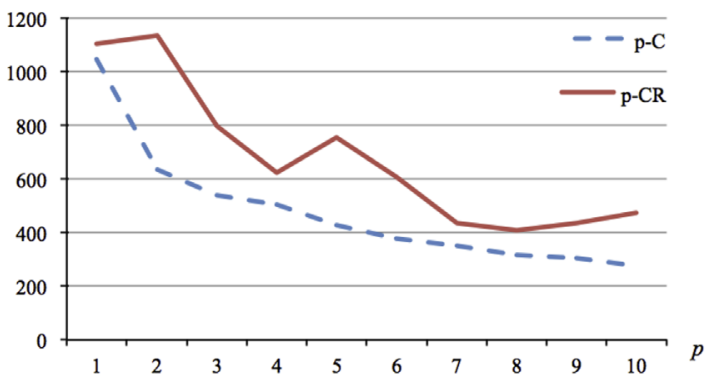

e

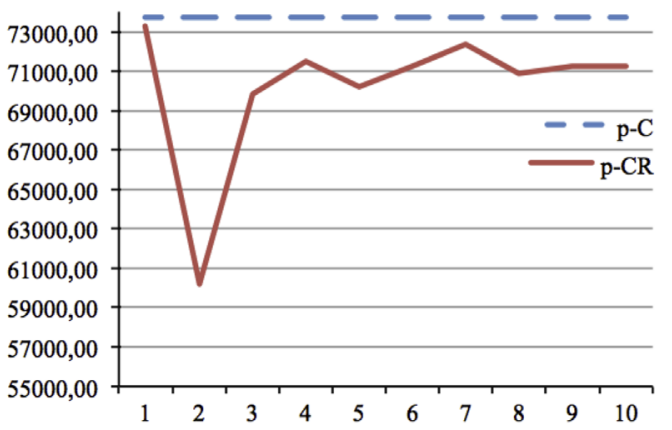

b

\section{weighted case}

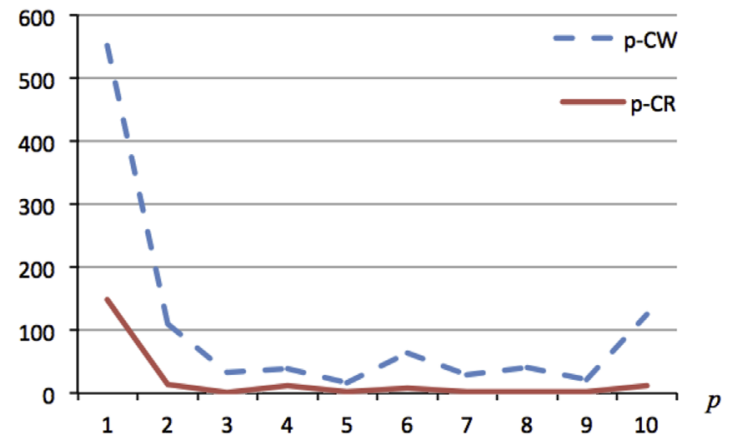

d

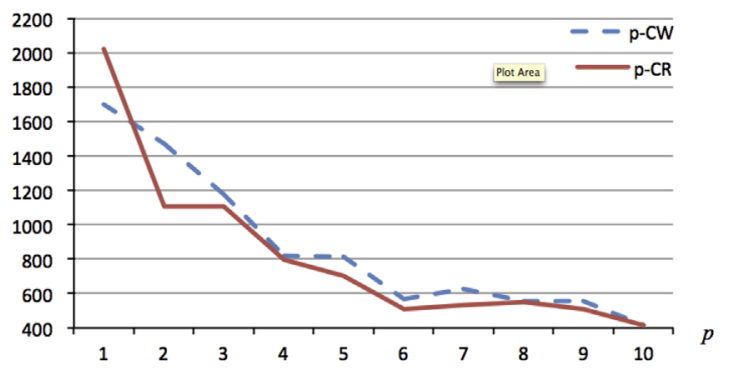

f

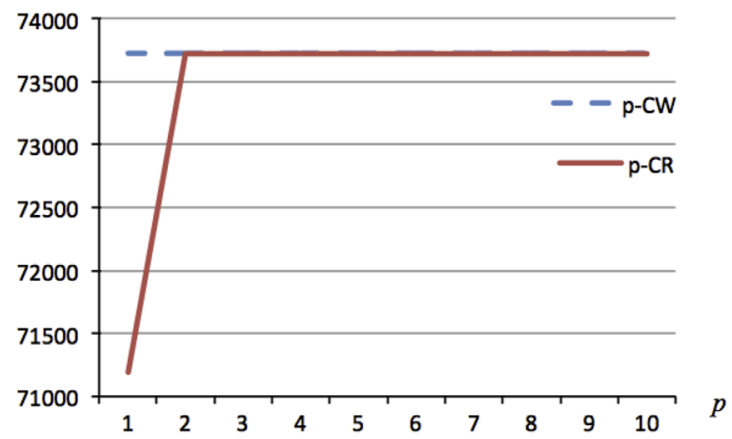

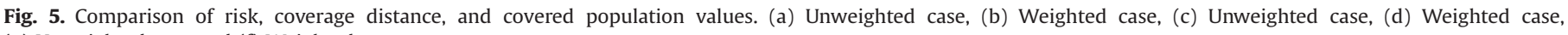
(e) Unweighted case and (f) Weighted case.

values throughout the network as well the number of facilities to locate and coverage distances. For example, for $p=1$, the facility is located in Kırklareli neighboring İstanbul. Kırklareli is located in the north-west of Turkey and has the smallest disruption probability among all locations. Because the west part of Turkey is more highly populated than the east part, the model locates the facility in Kirklareli and prefers not to cover demand points with low population in the east part. This is why the coverage distance $K^{p-C R}$ is higher than $K^{p-C W}$. The percentage of the population not covered in this case is $3.4 \%$ of the whole population. The location with the second lowest disruption probability is Aksaray (almost in the center of Turkey). If the model were to select that location, all demand points would be covered. However, this would increase the maximum risk value of İstanbul and hence that of the model. For $p=2, p-C R$ selects two neighboring locations Aksaray and Niğde, both of which have the second lowest disruption probabilities and are almost in the center of Turkey. Both locations cover all demand points with a coverage distance $K^{p-C R}$ lower than $K^{p-C W}$. The vulnerability of each demand point is the same and equal to the multiplication of the disruption probabilities. The resulting maximum value is again determined by İstanbul. However, notice that Kurklareli with the minimum disruption probability is not selected because in that case the risk value increases. When Kırklareli is selected as one of the locations, some demand points are not covered twice resulting in higher vulnerability values. One of those demand points is highly populated Diyarbakır in the southeastern part of Turkey and in the second degree earthquake zone. Its risk value becomes greater than that of İstanbul. This analysis also indicates that the model can make the aformentioned trade-offs correctly.

We remark that the resulting $K^{p-C R}$ and $C^{p-C R}$ may change for alternative optimal solutions. For example, Table 1 indicates that a risk value of 59,568 is obtained with $K^{p-C R}=408$ and $C^{p-C R}=70,862,590$ when $p=8$. However, another solution with the same risk value gives $K^{p-C R}=630$ and $C^{p-C R}=68,469,173$. So, it may be a good practice to search for alternative solutions with better population coverages and coverage distances. 


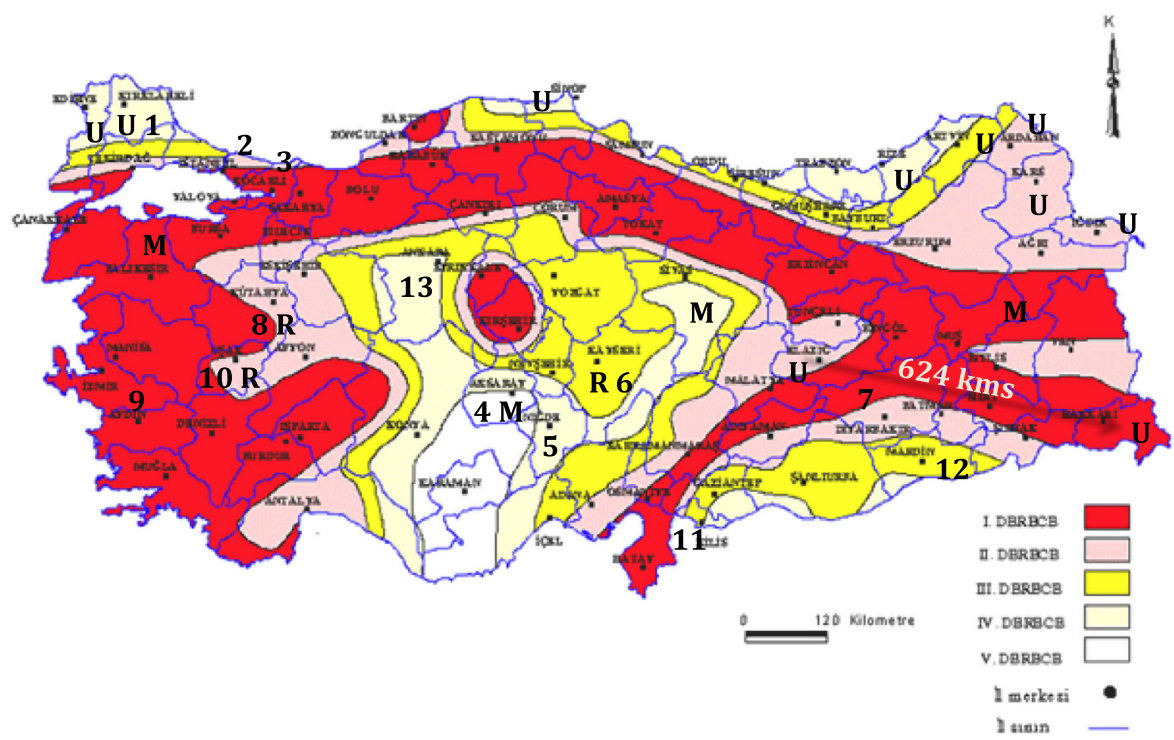

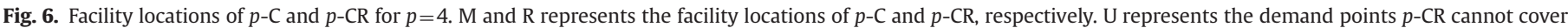

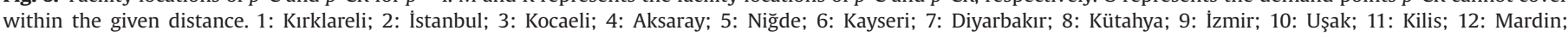
13: Ankara.

Table 3

Facility locations of $p-C R$ and $p-C$. R and $C$ stand for $p-C R$ and $p-C$, respectively.

\begin{tabular}{|c|c|c|c|c|c|c|c|c|c|c|}
\hline \multirow[t]{2}{*}{ Location } & \multicolumn{10}{|l|}{$p$} \\
\hline & 1 & 2 & 3 & 4 & 5 & 6 & 7 & 8 & 9 & 10 \\
\hline Kirklareli & & $\mathrm{R}$ & & & & $\mathrm{R}$ & $\mathrm{R}$ & $\mathrm{R}$ & $\mathrm{R}$ & $\mathrm{R}$ \\
\hline Uşak & & & & $\mathrm{R}$ & $\mathrm{C}$ & $\mathrm{R}$ & $\mathrm{R}$ & $\mathrm{R}, \mathrm{C}$ & $\mathrm{R}, \mathrm{C}$ & \\
\hline Balikesir & & & & C & $\mathrm{R}$ & & & & $\mathrm{R}$ & $\mathrm{R}$ \\
\hline Elaziğ & & C & & $\mathrm{R}$ & & & & & & \\
\hline Kayseri & $\mathrm{C}$ & & & & & & & $\mathrm{C}$ & & \\
\hline Kilis & & & & & & $\mathrm{R}$ & $\mathrm{R}$ & $\mathrm{R}$ & $\mathrm{R}$ & $\mathrm{R}$ \\
\hline Niğde & $\mathrm{R}$ & & & & $\mathrm{R}$ & & & & $\mathrm{C}$ & \\
\hline Kütahya & & & $\mathrm{R}$ & $\mathrm{R}$ & $\mathrm{R}$ & & & & & \\
\hline Erzurum & & & & & & & & $\mathrm{C}$ & $\mathrm{C}$ & $\mathrm{C}$ \\
\hline Osmaniye & & & & & & & $\mathrm{C}$ & $\mathrm{C}$ & $\mathrm{C}$ & \\
\hline
\end{tabular}

Table 4

Facility locations of $p-\mathrm{CR}$ and $p-\mathrm{CW}$. R and $\mathrm{W}$ stand for $p$-CR and $p-\mathrm{CW}$, respectively.

\begin{tabular}{|c|c|c|c|c|c|c|c|c|c|c|}
\hline \multirow{2}{*}{ Location } & \multicolumn{10}{|l|}{$p$} \\
\hline & 1 & 2 & 3 & 4 & 5 & 6 & 7 & 8 & 9 & 10 \\
\hline Kirklareli & $\mathrm{R}$ & & & $\mathrm{R}$ & $\mathrm{R}$ & $\mathrm{R}$ & & & $\mathrm{R}$ & \\
\hline Kütahya & & & & & & & $\mathrm{R}$ & $\mathrm{R}$ & $\mathrm{R}$ & $\mathrm{R}$ \\
\hline Niğde & & $\mathrm{R}$ & $\mathrm{R}$ & & & $\mathrm{R}$ & & $\mathrm{R}$ & & $\mathrm{R}$ \\
\hline Diyarbakir & & & & & $\mathrm{R}$ & $\mathrm{R}$ & & & $\mathrm{R}$ & \\
\hline Mardin & & & & $\mathrm{R}$ & $\mathrm{R}$ & & $\mathrm{R}$ & $\mathrm{R}$ & $\mathrm{R}$ & \\
\hline Konya & & & & & $\mathrm{R}$ & $\mathrm{R}$ & & & $\mathrm{R}, \mathrm{W}$ & \\
\hline Uşak & & & & & & $\mathrm{R}$ & $\mathrm{R}$ & $\mathrm{R}$ & & $\mathrm{R}$ \\
\hline Aksaray & & $\mathrm{R}$ & $\mathrm{R}$ & $\mathrm{R}$ & $\mathrm{R}$ & & & & & \\
\hline Ankara & & & & W & W & $\mathrm{R}, \mathrm{W}$ & $\mathrm{R}, \mathrm{W}$ & $\mathrm{R}$ & W & W \\
\hline İzmir & & & W & & & $\mathrm{W}$ & $\mathrm{W}$ & & & \\
\hline Osmaniye & & & & & W & W & W & & & W \\
\hline Antalya & & & & & & W & & & W & W \\
\hline Kocaeli & $\mathrm{W}$ & W & & & & & $\mathrm{R}$ & & W & $\mathrm{R}$ \\
\hline Manisa & & & & & & & & W & W & W \\
\hline İstanbul & & & W & W & W & W & W & W & W & $\mathrm{W}$ \\
\hline
\end{tabular}

\subsection{Facility locations}

Table 3 (Table 4 ) presents the facility locations selected by $p-C R$ and $p-\mathrm{C}(p-\mathrm{CW})$ at least 3 times for $p$ changing from 1 to 10 . The results indicate that the facility locations of $p-C$ and $p-C R(p-C W)$ are different except for a few cases. This is actually evident from the risk values discussed previously; if the facility locations were mostly common, the risk values would be close.

These tables are important because they indicate the locations to focus on. For example, Table 3 indicates that Kırklareli, Uşak, and Kilis are selected 6, 5, and 5 times, respectively, and Table 4 indicates that Kırklareli, Niğde, and Mardin are selected 5 times and Kütahya, Aksaray, and Uşak are selected 4 times. So, these locations are of critical importance and good candidates for locating facilities. Why Kırklareli is selected has been discussed in the preceding section. Uşak is in the center of the western part of Turkey in the first earthquake zone and has the lowest disruption probability in the region. İzmir with the highest value in the region can be covered by Uşak to minimize the risk value. Niğde and Aksaray neighbor each other and have the same and the second lowest disruption probability. They are in the center of Turkey and close to the capital of Turkey, Ankara, which is in the first earthquake zone and has the second highest population in Turkey. Mardin is in the eastern part of Turkey and close to demand points in the first earthquake zone as well as to Diyarbakır with the highest population in the region. Mardin has the lowest disruption probability in the region. Similar explanations hold for Kilis as well. We remark that İstanbul that has been selected by $p$-CW 8 times is not chosen by $p$-CR because İstanbul is in the first earthquake zone and has significantly large disruption probability. The other cases may be analyzed similarly. The results indicate the facility locations selected by $p$-CR are certainly more resilient than the ones selected by $p-C$ and $p-C W$.

\subsection{The change in risk, covered population, and coverage distance in $\mathrm{p}$-CR based on $\mathrm{K}$}

To determine how the risk, covered population, and coverage distance $K^{p-C R}$ change in $p$-CR depending on the given coverage distance $K, p$-CR is solved for coverage distances changing from zero to 2200 by increments of 200 with $p=1$. The results are shown graphically in Fig. 7(a). The results indicate that the risk value monotonically decreases when coverage distance is increased. The marginal contribution of increasing coverage distance to minimize the risk value diminishes as expected. For example, when the coverage distance is changed from 200 to 
a

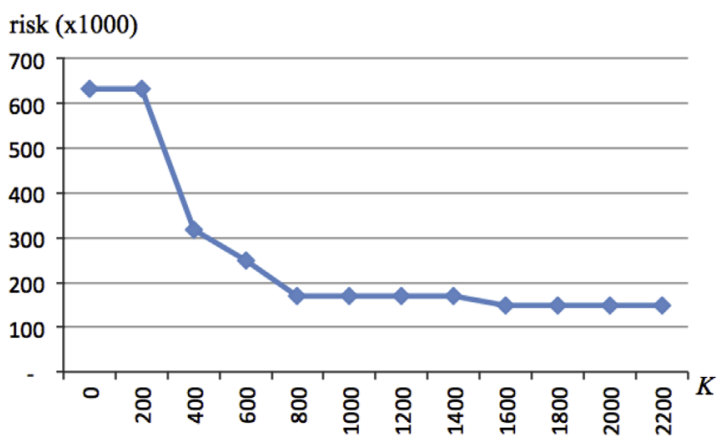

population $(\mathrm{x} 1000)$
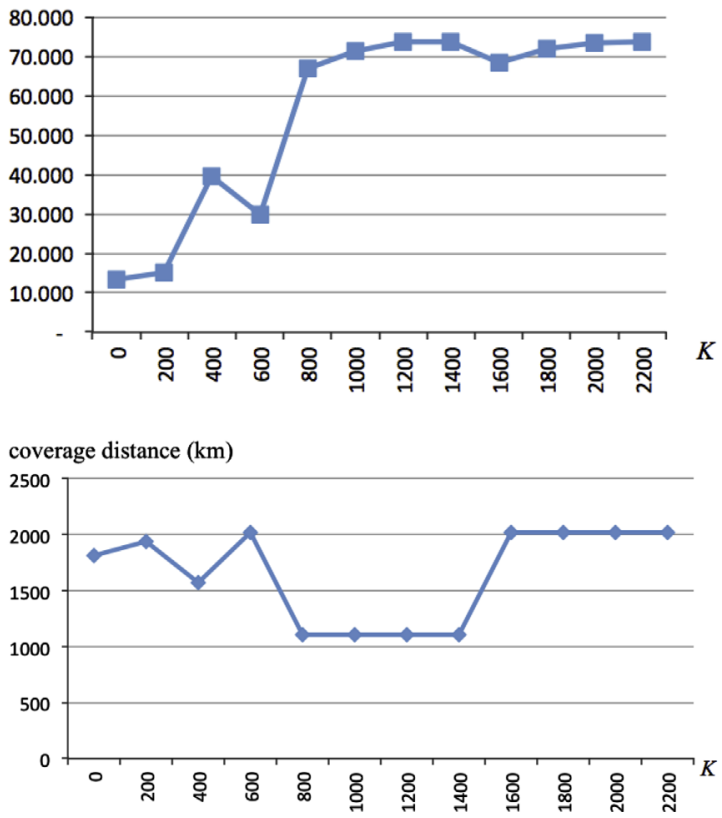

b

risk (x1000)

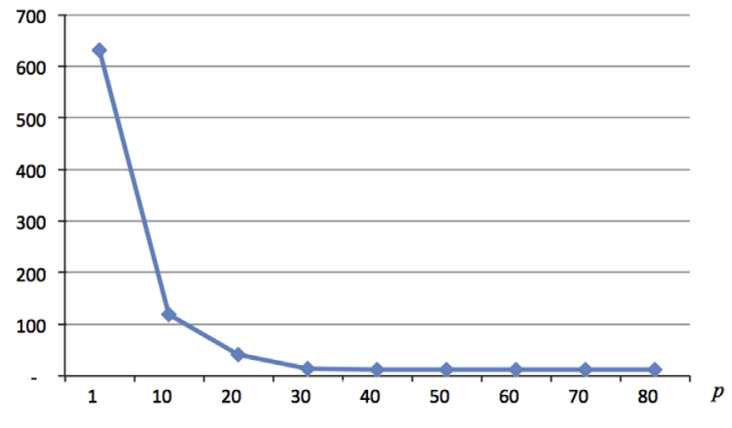

population $(\mathrm{x} 1000)$

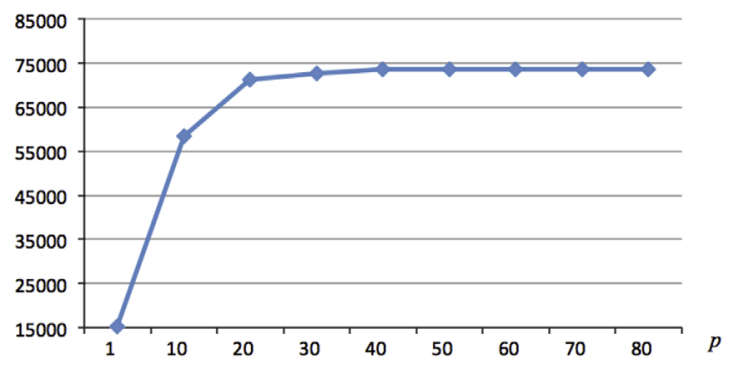

coverage distance $(\mathrm{km})$

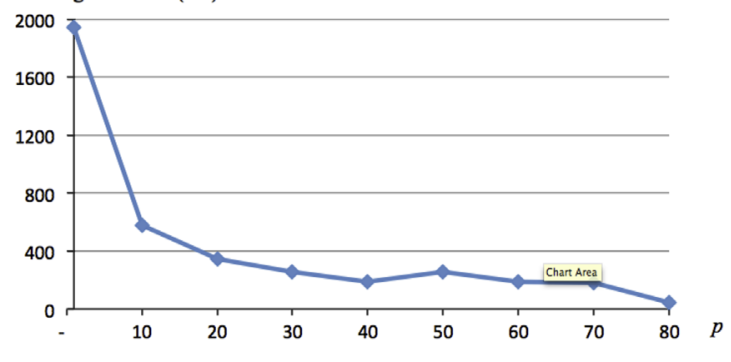

Fig. 7. Change in risk, covered population, and coverage distance based on (a) $K$ and (b) $p$.

400 , the decrease in the risk value is about $50 \%$. On the other hand, when the coverage distance is changed from 400 to 600 , the decrease is $21 \%$. Notice also that every increase in coverage distance does not necessarily mean a decrease in the risk value. For example, the model produces the same risk value for coverage distances ranging from 800 to 1400 and from 1600 to 2200. This result occurs because the facility is located at the same location and the same point determines the maximum risk value for those coverage distances.

Unlike risk value, population coverage does not follow a monotonic pattern even though there is an increasing trend. As long as the facility location does not change, the population coverage increases with increasing coverage distance as expected. For example, from 0 to 200, from 800 to 1400 , and from 1600 to 2200 , the facility location does not change but the population coverage increases. When the facility location changes, the population coverage may increase or decrease. For example, when coverage distance is increased from 200 to 400, better risk value and better population coverage are obtained simultaneously. On the other hand, population coverages decrease when the coverage distance is increased from 400 to 600 and from 1400 to 1600 . At these coverage distances (600 and 1600), the facility is placed at the locations that produce lower population coverages but better risk values. So, risk value is improved at the expense of population coverage.

With regard to coverage distance $K^{p-C R}$, no monotonic pattern is observed. $K^{p-C R}$ may decrease or increase depending on the facility location that may change with increasing $K$. As long as the location does not change, coverage distance $K^{p-C R}$ remains the same. Because the location alternatives increase with increasing coverage distance $K$, better risk values may be obtained. However, that does not necessarily mean better $K^{p-C R} . K^{p-C R}$ may be greater or less than $K$ depending on the location.

8.5. The change in risk, covered population, and coverage distance in $p$-CR based on $p$

To determine how the risk, covered population, and coverage distance $K^{p-C R}$ change in $p$-CR depending on $p$, the number of facilities to locate, $p$-CR is solved for $p$ changing from 1 to 80 with increments of 10 with $K=200$. The results are shown graphically in Fig. 7(b). The results indicate that the risk value decreases monotonically with increasing $p$. The marginal contribution of increasing $p$ diminishes as $p$ gets larger. The risk value decreases $81 \%, 66 \%, 66 \%$, and $12 \%$ when $p$ changes from 1 to 10 , from 10 to 
20 , from 20 to 30 , and from 30 to 40 , respectively. For $p \geq 40$, the risk value does not change at all.

With regard to population coverage, it increases monotonically with increasing $p$. For $p \geq 50$, the whole population is covered. For $p=40$, about $99.7 \%$ of the whole population is covered.

Coverage distance $K^{p-C R}$ decreases monotonically with diminishing return as $p$ is increased. It stabilizes for $p \geq 40$.

When all the results are considered, for $K=200, p=40$ may be considered as a good decision. If a larger coverage distance is used, $p$ will decrease accordingly. Actually, the risk value and population coverage obtained with $p=40$ and $p=30$, respectively, may be achieved with $p=10$ when $K=400$. Similarly, coverage distance $K^{p-C R}$ obtained with $p=20$ may be realized with $p=10$.

\section{An application of $p$-CR}

$p$-CR has been applied to suggest the locations of facilities that will be used by Turkish Red Crescent Society (TRC) for prepositioning disaster-relief items. The underlying structure defined in the previous section has been used after the confirmation by TRC. TRC has currently split Turkey into 10 service regions and located a facility for each region. Because of a need to reorganize, it has started a decision making process to revise the number and locations of the facilities. It has considered three decision alternatives: (1) current 10 facilities, (2) 15 facilities, and (3) 16 facilities. $p$-CR has been developed after the decision process has been initiated and decision alternatives have almost been finalized. In this context, $p$-CR has been used to evaluate the planned locations and to suggest new locations in three steps: (1) the risk values $R^{\text {Planned }}$ and coverage distances $K^{\text {Planned }}$ of the planned locations have been determined, (2) $p$-CR has been used to determine facility locations with coverage distances $K=K^{\text {Planned }}$ for $p=10,15$, and 16 , and (3) the current 10 facility locations have been fixed and new locations have been determined by $p-C$ and $p$-CR for $p$ changing from 11 to 16 with $K=K^{p-C}$.

The risk values, coverage distances, and population coverages are given Table 5. The results indicate that the differences between the risk values of plannned locations and those of $p$-CR are significant. The risk values of planned locations are higher than those of $p$-CR about 19, 16, and 30 times for $p=10,15$, and 16 , respectively. For all three cases, $p$-CR covers the whole population with a smaller coverage distance.

When the locations of $p$-CR are considered, there is one common location for $p=10$ and two common locations for $p=15$ and 16 with those of the planned locations. On the other hand,

Table 5

The results for the application.

\begin{tabular}{|c|c|c|c|c|c|c|}
\hline \multirow[t]{2}{*}{$p$} & \multicolumn{3}{|l|}{ Planned } & \multicolumn{3}{|l|}{$p-C R$} \\
\hline & $R^{\text {Planned }}$ & $K^{\text {Planned }}$ & $C^{\text {Planned }}$ & $R^{p-C R}$ & $K^{p-C R}$ & $C^{p-C R}$ \\
\hline 10 & 166,067 & 428 & $73,722,988$ & 8854 & 341 & $73,722,988$ \\
\hline 15 & 83,139 & 360 & $73,722,988$ & 5141 & 359 & $73,722,988$ \\
\hline 16 & 68,698 & 395 & $73,722,988$ & 2296 & 371 & $73,722,988$ \\
\hline \multirow[t]{2}{*}{$p$} & \multicolumn{3}{|l|}{$p-C$} & \multicolumn{3}{|l|}{$p-C R$} \\
\hline & $R^{p-C}$ & $K^{p-C}$ & $C^{p-C}$ & $R^{p-C R}$ & $K^{p-C R}$ & $C^{p-C R}$ \\
\hline $10+1$ & 166,067 & 428 & $73,722,988$ & 72,565 & 428 & $73,722,988$ \\
\hline $10+2$ & 166,067 & 395 & $73,722,988$ & 39,084 & 428 & $73,520,248$ \\
\hline $10+3$ & 166,067 & 371 & $73,722,988$ & 40,208 & 395 & $73,268,950$ \\
\hline $10+4$ & 166,067 & 320 & $73,722,988$ & 40,208 & 403 & $73,268,950$ \\
\hline $10+5$ & 166,067 & 294 & $73,722,988$ & 33,235 & 403 & $73,520,248$ \\
\hline $10+6$ & 166,067 & 275 & $73,722,988$ & 40,208 & 395 & $72,851,890$ \\
\hline
\end{tabular}

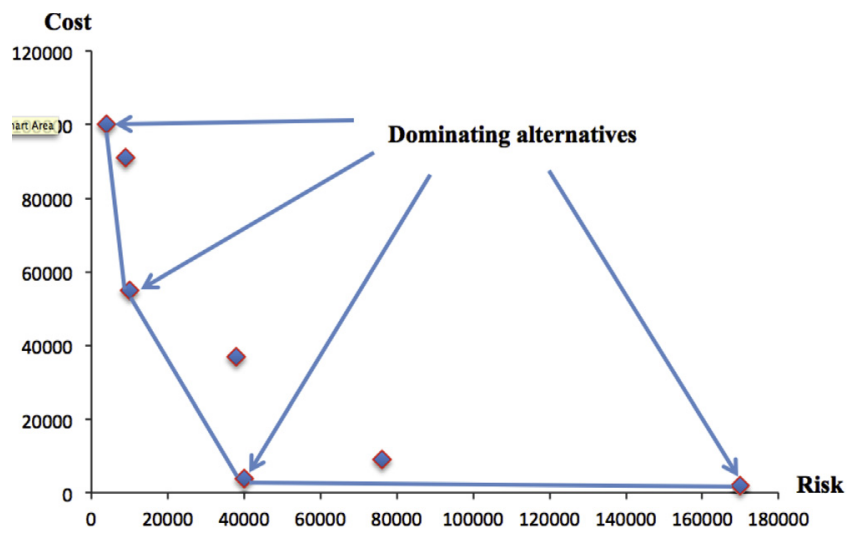

Fig. 8. Alternatives on the objective value space (risk and cost).

almost all of the locations given in Table 3 are selected by $p$-CR as expected.

The results with 10 fixed locations indicate that the locations determined by $p-C$ cannot improve the rerefence risk value with $p=10$ even though the coverage distances are improved. On the other hand, $p$-CR is able to find solutions with better risk values. However, the improvement upon the reference solution is limited; the risk value can be decreased up to about $25 \%$ of the current solution. Actually, the risk values between $p=10+3$ and $p=10+6$ are almost the same. In this regard, because more than $99 \%$ of the population is covered for all cases even though the coverage distances are not the same, locating $10+3$ may be a costeffective decision. On the other hand, it is clear that much better risk values are realized when all facility locations are determined from scratch. However, this incurs much higher costs.

To present the results in a structured way, we have represented the alternatives on the objective value space on a graph similar to the one given in Fig. 8 with the axes representing the risk and cost values. From the graph, we have determined the dominating alternatives and explained which dominating alternative is to be chosen depending on the preference of the decision maker. TRC has not made the decision yet but $p$-CR clearly has added a new, valuable dimension to take into account.

\section{Conclusion}

In this study, we address the facility location problem in the pre-disaster phase of DM operations. We develop an optimization model that minimizes the risk that a demand point may be exposed to because it is not supported by the located facilities. The purpose is to choose the locations such that a reliable facility network to support the demand points is constructed. The risk for a demand point is measured as the multiplication of the (probability) threat, the vulnerability of the demand point, and the consequence. The vulnerability of a demand point is computed by using fault tree analysis and incorporated into the optimization model innovatively. To our knowledge, this paper is the first to use such an approach. The resulting model is tested with several problems. The results indicate that taking the risk into account explicitly may create significant differences in the risk levels.

\section{Acknowledgments}

The authors are grateful to two anonymous referees for providing constructive feedback that has helped improve in major ways the presentation of the material in the paper. 


\section{References}

[1] Schulz SF. Disaster relief logistics: benefits of and impediments to horizontal cooperation between humanitarian organizations [Ph.D. Thesis]. Technische Universitat Berlin, 2008.

[2] McLoughlin D. A framework for integrated emergency management. Public Administration Review (Special Issue: Emergency Management: A Challenge for Public Administration) 1985(45):165-72.

[3] Altay N, Green WG. OR/MS research in disaster operations management. European Journal of Operational Research 2006;175(1):475-93.

[4] Coppola DP. Introduction to international disaster management. ButterworthHeinemann; 2011.

[5] Asian Disaster Preparedness Center (ADPC), Bangkok under Capacity Building in Asia Using Information Technology Applications (CASITA), Course Notes. Available from 〈http://www.adpc.net/casita/course-materials/Mod-4-Disas ter-Mgmt.pdf $\rangle$ [Accessed on 02.08. 2013].

[6] United Nations International Strategy for Disaster Reduction, Hyogo Framework for Action 2005-2015, World Confence on Disaster Reduction, 18-22 Jan 2005, Hyogo, Japan. Available from 〈http://www.unisdr.org/2005/wcdr/ wcdr-index.htm $\rangle$ [Accessed 02.08. 2013].

[7] Balcik B, Beamon BM. Facility location in humanitarian relief. International Journal of Logistics: Research and Applications 2008;11(2):101-21.

[8] Jahre M, Heigh I. Does the current constraints in funding promote failure in humanitarian supply chains? Supply Chain Forum: An International Journal 2008;9(2):44-54.

[9] Thomas A, Mizushima M. Logistics training: necessity or luxury? Forced Migration Review 2005;22:60-1.

[10] Galindo G, Batta R. Review of recent developments in OR/MS research in disaster management. European Journal of Operational Research 2013;230 (2):201-11.

[11] Caunhye AM, Nie X, Pokharel S. Optimization models in emergency logistics: literature review. Socio-Economic Planning Sciences 2012;46(1):4-13.

[12] Jia H, Ordóñez F, Dessouky M. A modeling framework for facility location of medical services for large-scale emergencies. IIE Transactions 2007;39 (1):41-55.

[13] Jia H, Ordóñez F, Dessouky MM. Solution approaches for facility location of medical supplies for large-scale emergencies. Computers \& Industrial Engineering 2007;52(2):257-76.

[14] Dessouky M, Ordóñez F, Jia H, Shen Z. Rapid distribution of medical supplies. In: Hall R, editor. Patient flow: reducing delay in healtcare delivery. New York: Springer; 2006. p. 309-38.

[15] Belardo S, Harrald J, Wallace WA, Ward J. A partial covering approach to siting response resources for major maritime oil spills. Management Science 1984:30(10):1184-96.

[16] Charnes A, Cooper WW, Harrald J, Karwan KR, Wallace WA. A goal interval programming model for resource allocation in a marine environmental protection program. Journal of Environmental Economics and Management 1976;3(4):347-62.

[17] McCall VM. Designing and prepositioning humanitarian assistance pack-up kits (HA PUKs) to support pacific fleet emergency relief operations [Master's Thesis]
Department of Operations Research, Naval Postgraduate School, Monterrey, CA, 2006.

[18] Wilhelm WE, Srinivasa AV. A strategic, area-wide contingency planning model for oil spill cleanup operations with application demonstrated to the Galveston Bay area. Decision Sciences 1996;27(4):767-99.

[19] Duran S, Gutierrez MA, Keskinocak PN. Pre-positioning of emergency items worldwide for CARE international. Interfaces 2011;41(3):223-37.

[20] Rawls CG, Turnquist MA. Pre-positioning of emergency supplies for disaster response. Transportation Research Part B: Methodological 2010;44(4):521-34.

[21] Daskin MS. A maximum expected covering location model: formulation, properties and heuristic solution. Transportation Science 1983;17(1):48-70.

[22] Drezner Z. Heuristic solution methods for two location problems with unreliable facilities. Journal of the Operational Research Society 1987;38 (6):509-14.

[23] Lee SD. On solving unreliable planar location problems. Computers and Operations Research 2001:28:329-44.

[24] Snyder LV, Daskin MS. Reliability models for facility location: the expected failure cost case. Transportation Science 2005;39(3):400-16.

[25] Berman O, Krass D, Menezes MBC. Facility reliability issues in network p median problems: strategic centralization and co-location effects. Operations Research 2007;55(2):332-50.

[26] Berman O, Krass D, Menezes MBC. Location and reliability problems on a line: impact of objectives and correlated failures on optimal location patterns. OMEGA 2013;41(4):766-79.

[27] Ukkusuri SV, Yushimito WF. Location routing approach for the humanitarian prepositioning problem. Transportation Research Record: Journal of the Transportation Research Board 2008;2089:18-25.

[28] Campbell AM, Jones PC. Prepositioning supplies in preparation for disasters. European Journal of Operational Research 2011;209(2):156-65.

[29] Willis HH, Morral AR, Kelly TK, Medby JJ. Estimating terrorism risk. Santa Monica, CA: RAND Corporation; 2005 (MG-388-OSD).

[30] Rahman FA, Varuttamaseni A, Kintner-Meyer M, Lee JC. Application of fault tree analysis for customer reliability assessment of a distribution power system. Reliability Engineering \& System Safety 2013;111:76-85.

[31] Zhang M, Kecojevic V, Komljenovic D. Investigation of haul truck-related fatal accidents in surface mining using fault tree analysis. Safety Science 2014;65 (1-6-117).

[32] Vesely WE, Goldberg FF, Roberts NH, Haasl DF. Fault tree handbook. Washington, DC: U.S. Nuclear Regulatory Commission; 1981.

[33] Lu C-C, Sheu J-B. Robust vertex p-center model for locating urgent relief distribution centers. Computers and Operations Research 2013:40(8):2128-37.

[34] Bell JE, Griffis SE, Cunningham III WA, Eberlan JA. Location optimization of strategic alert sites for homeland defense. OMEGA 2011;39(2):151-8.

[35] Hakimi SL. Optimum distribution of switching centers in a communication network and some related graph theoretic problems. Operations Research $1965 ; 13: 462-75$

[36] GAMS Development Corporation, GAMS-A user's guide, Washington, DC, 2013.

[37] IBM Corporation, IBM ILOG CPLEX V12.1 User's manual for CPLEX, 2009.

[38] Gökçe O, Özden Ş, Demir A. An information inventory of the locational and statistical distribution of disasters in Turkey, Ministry of Public Works and Settlement, General Directorate of Disaster Affairs, Ankara, 2008 [in Turkish]. 